\title{
Dynamic real-time capacity constrained routing algorithm for evacuation planning problem
}

\author{
Jawad Abusalama ${ }^{1}$, Sazalinsyah Razali ${ }^{2}$, Yun-Huoy Choo $^{3}$, Lina Momani ${ }^{4}$, \\ Abdelrahman Alkharabsheh ${ }^{5}$ \\ ${ }^{1,2,3}$ Faculty of Information and Communication Technology, Universiti Teknikal Malaysia Melaka, Malaysia \\ ${ }^{4}$ Faculty of Engineering Technology and Science, Higher Colleges of Technology, UAE \\ ${ }^{5}$ Faculty of Engineering and Computer Sciences, Mustaqbal University, KSA
}

\begin{tabular}{l}
\hline \hline Article Info \\
\hline Article history: \\
Received Sep 6, 2019 \\
Revised Jan 28, 2020 \\
Accepted May 6, 2020 \\
\hline
\end{tabular}

\section{Keywords:}

Emergency route planning disasters

Evacuation plan

Evacuation planning problem

Heuristic algorithm

\begin{abstract}
Usually, disasters occur over a relatively short time anytime and anywhere. Most occupancies do not have absolute knowledge about the prevention or safety consciousness to deal with disasters. During disaster occurrence, evacuation processes are conducted to save people's life, and if there is no appropriate evacuation plan, the situation will become worse. Thus, finding an optimal planning technique to evacuate occupants is critical in many cases i.e. emergency evacuation. In this paper, a Dynamic Real-Time Capacity Constrained Routing (DRTCCR) Algorithm has been proposed and analyzed. Such algorithm will investigate the capacity constraints of the evacuation network in real time by modelling the capacities based on time series to improve current solutions of the Emergency Route Planning (ERP) problem. Such algorithm will produce an optimal solution for the ERP problem. Performance evaluation on many network models illustrates that the DRTCCR algorithm improves the previous evacuation planning by reducing the evacuation time as well as the computational cost. In addition, DRTCCR algorithm has the ability to recalculate and find out the optimal path dynamically in real time irrespective of the number of trapped people as well as the transportation network size. Analytical experiments have been carried out, which illustrates the efficiency of the proposed algorithm.
\end{abstract}

Copyright $(2020$ Institute of Advanced Engineering and Science. All rights reserved.

\section{Corresponding Author:}

Jawad Abusalama,

Faculty of Information and Communication Technology,

Universiti Teknikal Malaysia Melaka, Malaysia.

Email: jenin2101@gmail.com

\section{INTRODUCTION}

In recent years, the amount in the occurence of disasters has risen from 50 to 400 per year and it is still expected to increase five times more in the next 50 years [1]. A natural disaster is a sudden event that occurs due to natural forces of the Earth or Human faults, leading to the destruction and killing of numerous persons [2]. Emergency can be defined as a status that requires a critical time response caused by a catastrophic phenomenon, which could be natural (i.e. earthquake, flood and hurricane) or human-made (i.e. Hazardous materials, fire), and that could put human lives at risk [3]. In case of disaster occurrence, it is difficult for people at risk to be evacuated in a smooth manner. However, it is not easy to understand the situation because people at risk often become scared in the course of a disaster. In addition, the rescuing corridors in buildings or squares become congested with the people [4].

Depending on the disaster type, the pre-warning of sudden-onset disasters can provide enough time for evacuations prior to the event [5]. Another influential factor in the case of natural disasters is the 
probability of their occurrence and effects. Thus, to reduce the effects of the disaster, it is necessary to take many actions before and during the disaster occurence i.e. transportation management; provide safe emergency exits for people at risk and ensure the availability of clear optimal evacuation plans during the disaster occurence. Furthermore, during disaster occurrence, people at risk need to know the optimal routes that can be used for evacuation. In addition, it is essential to have knowledge on how to react to unexpected events at the initial stage of planning [6]. Evacuation planning can be defined as a risk management strategy that would minimize loss of life or reduce the effects of disaster before and during the occurrence of a disaster [7]. Therefore, evacuation planning should be designed and implemented.

Several algorithms have been implemented the evacuation planning problem to finding the optimal Emergency Routes. Such that generic algorithm [8], polynomial algorithm [9], linear programming (LP) [10-12], cellular automata (CA) algorithm [13, 14], Immune Algorithm [3, 15] and neural algorithm [16]. Where, these methods gets the optimal Emergency Routes planning by using the timeexpanded graph structure. On the other hands, one of the popular method for solving the evacuation planning problem is the heuristic approaches method [17-20], that aims at producing a suboptimal evacuation route planning based on the transportation network, instead of the time-expanded graph structure. In the heuristic method, they take into consideration the route capacity constraints, and there are some constraints in this method, such as complexity of transportation networks, as well as largness in the number of evacuees, which leads to the production of inefficient evacuation plans. Therefore, many stuies have been done to deal with such constraints, such that Lu et al. [18] proposed a heuristic iterative algorithm Capacity Constrained Route Planner (CCRP) that produces a suboptimal solution for the evacuation planning problem. The static network has been used to minimize the computational cost for the time-expanded network. CCRP ensures that all evacuees were completely evacuated, but the main drowbaks of such algorithm is the produced evacuation paths allow intersection nodes to hold flow for some period of time [21]. In addition, Guo et al. [17] also proposed a heuristic approach to solve the evacuation route planning problem, taking into consideration the capacity constrained. In his study, Max-Flow Rate Priority (MFRP) algorithm was designed to obtain multiple candidate routes with maximum flow rate frequently. Such algorithm updates the potential available capacity of the transportation network for the next iteration without taking into considerations the priority of the paths during the evacuation process.

Furthermore, Lu et al. [19] proposed a classical Capacity Constrained Route Planner algorithm, wherein two sub-algorithms were proposed; Single-Route Capacity Constrained Planner (SRCCP) and Multiple-Route Capacity Constrained Planner (MRCCP). One of the drawbacks of such method is the small size building networks which are used to evaluate the performance of such algorithms. Such method leads to the increase in the total evacuation time due to increased waiting time. To improve these disadvantages, an evacuation route algorithm has been proposed by Zeng and Wang [22], thus recommending a longer evacuation route preferential algorithm. In fact, the proposed algorithm works well for long evacuation routes in most cases, however, in shorter evacuation routes, this algorithm is inefficient [23].

Apparently, it is obvious that all previous work did not mention the selection of the best path for the second iteration dynamically in real time. Mainly, when calculating the evacuation times, more than one best path is available with a minimum wait at the same time [24, 25]. In this paper, the proposed algorithm deals with such issue by recalculating the best path for each iteration. Furthermore, the proposed algorithm improves the performance of previous algorithms by reducing the evacuation time as well as minimizing the computational cost.

\section{PROPOSED ALGORITHM}

The main purpose of Dynamic Real-Time Capacity Constrained Routing (DRTCCR) algorithm is to create an optimal dynamic evacuating plan to identify the Best Evacuation Path (BEP) that can evacuate the occupancy in case of disaster occurrence.

\subsection{Notations}

This section presents the mathematical notation which is used in the proposed algorithm as shown in Table 1. Static network $G=(N$; E) was considered to represent the transportation network in the area of interest.

\subsection{Proposed algorithm}

This section the main steps of the DRTCCR algorithm are listed in this subsection and the flowchart of the proposed algorithm as well, which is shown in Figure 1.

Step 1: Define a graph $G(\mathrm{~N} ; \mathrm{E})$ is the transportation network with a set of nodes $\mathrm{N}$ and a set of edges E. 
Step 2: Determine if $N$ is a source node ( $\mathrm{Sn})$

$\mathrm{Sn}=\{\mathrm{S} 1, \mathrm{~S} 2, . . \mathrm{Sn}\}, \mathrm{Sn} \subseteq \mathrm{N}$

For each (Sn), determine Occupancy

$\mathrm{Ks}=\{\mathrm{Ks} 1, \mathrm{Ks} 2 \ldots \mathrm{Ks} \mathrm{n}\}$

Step 3: Determine if $N$ is a destination node (Dn)

$\mathrm{Dn}=\{\mathrm{D} 1, \mathrm{D} 2, . . \mathrm{Dn}\}, \mathrm{Dn} \subseteq \mathrm{N}$

Step 4: Find all paths for each source node $(\mathrm{Sn})$

Ps $=\{$ Ps 1 , Ps 2, ..Ps $n\}$

Step 5: For each path (Ps) in each source nodes ( $\mathrm{Sn}$ ), determine: $\mathrm{Cp}$, Tp, and wp

Where

$$
\begin{array}{ll}
w p=T p / C p, & K s \geq C p \\
w p=T p / K ' s, & K s<C p
\end{array}
$$

Step 6: Determine the Best Evacuation Path (BEP)

$\mathrm{BEP}=\operatorname{Min}(\mathrm{wp} 1, \mathrm{wp} 2, \ldots \mathrm{wpn})$

*In a case where two or more (BEP) have the same minimum waits (wp,) use all of them at the same time.

$\mathrm{K}$ 's $=\mathrm{Ks}-\mathrm{Cp}$ (selected path)

While K', s $>0$ do

\{

For each time the selected path used

$\mathrm{Tp}=\mathrm{Tp}+\mathrm{tp}$ (selected path)

$\mathrm{K}^{\prime} \mathrm{s}=\mathrm{Ks}-\mathrm{Cp}$

$\mathrm{Ks}=\mathrm{K}$ 's;

\}

Go back to step 5 .

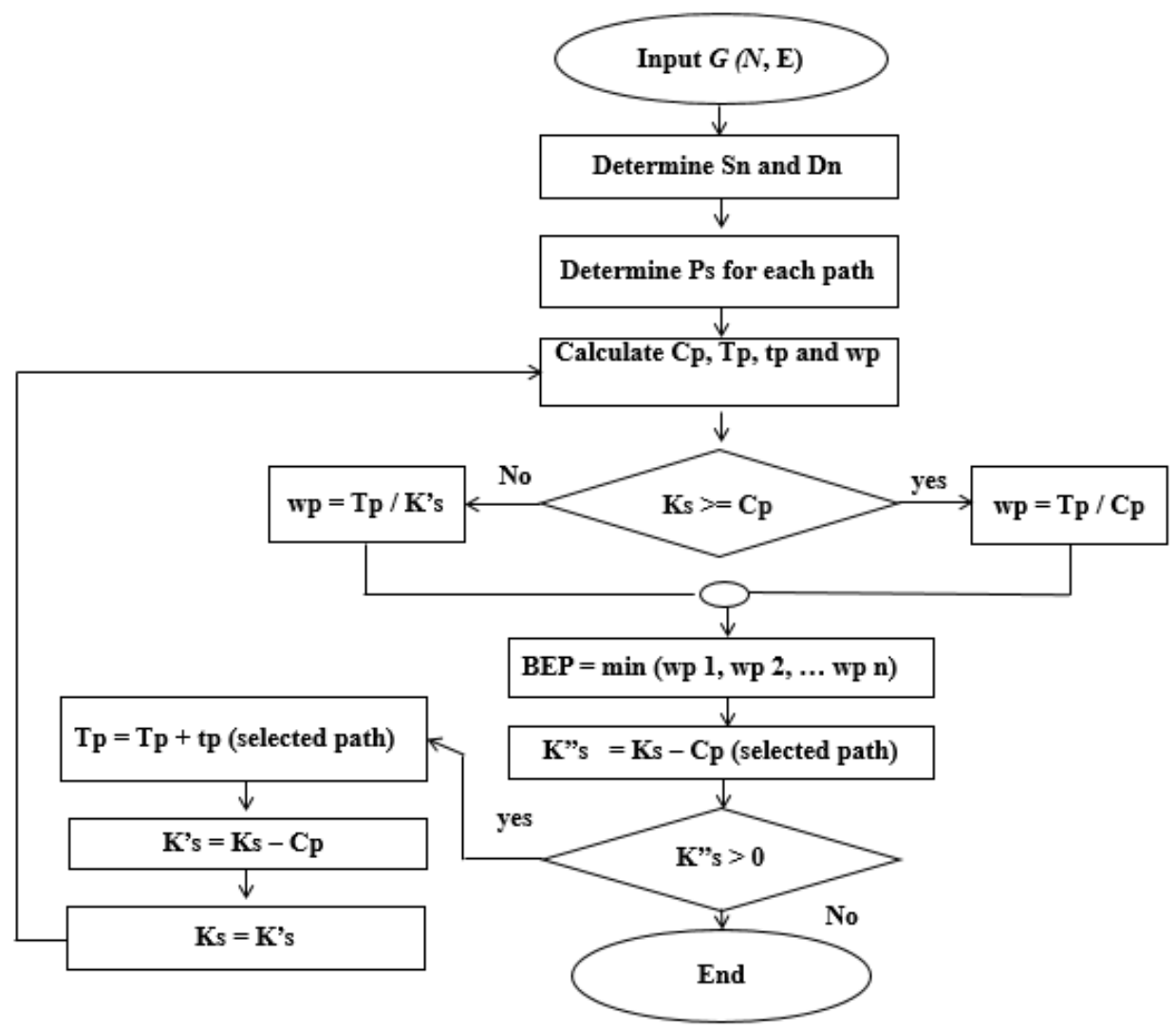

Figure 1. Flowchart of DRTCCR algorithm 
Table 1. Network notations

\begin{tabular}{cc}
\hline Notation & Description \\
\hline $\mathrm{N}$ & Set of Nodes \\
$\mathrm{E}$ & Set of Edge \\
$\mathrm{Sn}$ & Set of source nodes \\
$\mathrm{Dn}$ & Set of destination nodes \\
$\mathrm{Ps}$ & Evacuation paths of each source node \\
$\mathrm{Cp}$ & Minimum Capacity of each path \\
$\mathrm{Tp}$ & Total time of each path \\
tp & Delay time of each path $=1$ \\
$\mathrm{Ks}$ & Occupancy at source node \\
$\mathrm{K}$ 's & Remaining occupancy \\
K's & Check if all occupancy are evacuated \\
$\mathrm{wp}$ & The wait of each path \\
\hline
\end{tabular}

\subsection{Proposed algorithm evaluation}

To test and evaluate the proposed algorithm, it was applied on various transportation network models which were used for previous proposed algorithms. The transport network model of $\mathrm{Lu}$ et al. [19] has been used as shown in Figure 2. The presented transport network model composed of 15 nodes and 17 edges with its characteristics i.e. nodes capacity, initial occupancy for source nodes, edges capacity and the travel time for each edge. The flow of occupancy to be evacuated should travel from the source nodes $(\mathrm{N} 1, \mathrm{~N} 2$ and $\mathrm{N} 8)$ to the destination nodes (N13 and N14).

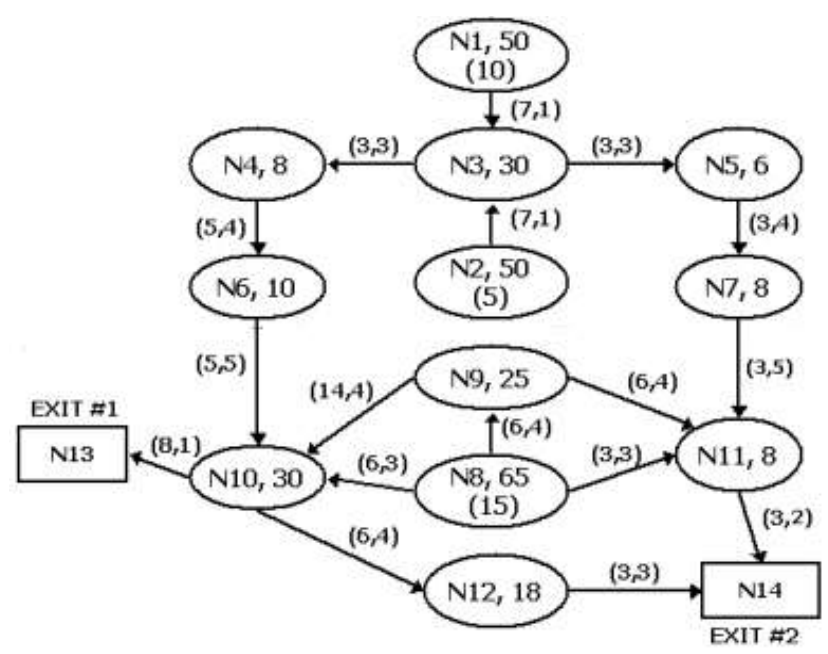

Figure 2. Transportation network of $\mathrm{Lu}$, et al. [19]

The presented steps of the DRTCCR algorithm as shown in section 2.2 are applied on the transportation network model which was shown in Figure 2.

Step 1: Define the transportation network.

Step 2: Determine the source nodes $(\mathrm{Sn})$ and the Occupants in each $(\mathrm{Sn})$.

$\mathrm{Sn}=\{\mathrm{N} 1, \mathrm{~N} 2$ and $\mathrm{N} 8\}$.

For (N1), the Occupancy (Ks 1) $=10$.

For (N2), the Occupancy (Ks 2) $=5$.

For (N8), the Occupancy (Ks 8) $=15$.

Step 3: Determine the destination nodes (Dn)

$\mathrm{Dn}=\{\mathrm{N} 13$ and N14 $\}$

Step 4: Find all paths (Ps) for each source node (Sn) as shown in Table 2.

Step 5: For each path (Ps) in each source nodes ( $\mathrm{Sn}$ ) determine: $\mathrm{Cp}$, Tp and wp.

Iteration 1: For $\mathrm{N} 1$, Where $\mathrm{Ks} \geqq \mathrm{Cp}, \mathrm{wp}=\mathrm{Tp} / \mathrm{Cp}$.

The values of $\mathrm{Cp}$, Tp and wp for each path (Ps) is shown in Table 3. 
Table 2. Selected paths for all nodes

\begin{tabular}{cl}
\hline Source nodes $(\mathrm{Sn})$ & \multicolumn{1}{c}{ Paths Ps } \\
\hline For N1 & Ps $1=\mathrm{N} 1, \mathrm{~N} 3, \mathrm{~N} 4, \mathrm{~N} 6, \mathrm{~N} 10, \mathrm{~N} 13$. \\
& Ps $2=\mathrm{N} 1, \mathrm{~N} 3, \mathrm{~N} 4, \mathrm{~N} 6, \mathrm{~N} 10, \mathrm{~N} 12, \mathrm{~N} 14$. \\
& Ps $3=\mathrm{N} 1, \mathrm{~N} 3, \mathrm{~N} 5, \mathrm{~N} 7, \mathrm{~N} 11, \mathrm{~N} 14$. \\
For N2 & Ps $1=\mathrm{N} 2, \mathrm{~N} 3, \mathrm{~N} 4, \mathrm{~N} 6, \mathrm{~N} 10, \mathrm{~N} 13$. \\
& Ps $2=\mathrm{N} 2, \mathrm{~N} 3, \mathrm{~N} 4, \mathrm{~N} 6, \mathrm{~N} 10, \mathrm{~N} 12, \mathrm{~N} 14$. \\
& Ps 3=N2, N3, N5, N7, N11, N14. \\
\cline { 2 - 2 } For N8 & Ps $1=\mathrm{N} 8, \mathrm{~N} 10, \mathrm{~N} 13$. \\
& Ps 2=N8, N10, N12, N14. \\
& Ps 3=N8, N9, N10, N13. \\
& Ps 4=N8, N9, N10, N12, N14. \\
& Ps $5=\mathrm{N} 8, \mathrm{~N} 9, \mathrm{~N} 11, \mathrm{~N} 14$. \\
& Ps 6=N8, N11, N14.
\end{tabular}

Table 3. The values of $\mathrm{Cp}$, Tp and wp for each path

\begin{tabular}{lccc}
\hline \multicolumn{1}{c}{ Path (Ps) } & Cp & Tp & wp \\
\hline Ps $(1)=$ N1, N3, N4, N6, N10, N13. & 3 & 14 & 4.66 \\
Ps (2) = N1, N3, N4, N6, N10, N12, N14. & 3 & 20 & 6.66 \\
Ps (3) = N1, N3, N5, N7, N11, N14. & 3 & 15 & 5 \\
\hline
\end{tabular}

Step 6: The Best Evacuation Path (BEP) is selected based on the minimum waiting time from all paths and it is Ps (1) with 4.66 waiting time.

To Check if all occupancies are evacuated or not, K's should be calculated as follow.

1. $\mathrm{K}$ 's $=\mathrm{Ks}-\mathrm{Cp}$ (selected path) $=10-3=7$ which is more than 0 .

2. While K's $>0$, Tp for selected path $=\mathrm{Tp}+\mathrm{tp}=15$.

3. $\mathrm{K}$ 's $=\mathrm{Ks}-\mathrm{Cp}=10-3=7$.

4. $\mathrm{Ks}=\mathrm{K}$ 's $=7$.

5. Go back to step 5, taking into considerations the new parameters values.

Iteration 2: Where $\mathrm{Ks} \geqq \mathrm{Cp}, \mathrm{wp}=\mathrm{Tp} / \mathrm{Cp}$.

The values of $\mathrm{Cp}$, Tp and wp for each path (Ps) is shown in Table 4.

Table 4. The values of $\mathrm{Cp}$, Tp and wp for each path

\begin{tabular}{lccc}
\hline \multicolumn{1}{c}{ Path (Ps) } & Cp & Tp & wp \\
\hline Ps $(1)=$ N1, N3, N4, N6, N10, N13. & 3 & 15 & 5 \\
Ps $(2)=$ N1, N3, N4, N6, N10, N12, N14. & 3 & 20 & 6.66 \\
Ps (3) = N1, N3, N5, N7, N11, N14. & 3 & 15 & 5 \\
\hline
\end{tabular}

The Best Evacuation Path (BEP) is selected based on the minimum waiting time from all paths and they are Ps (1) and Ps (3) with a waiting time of 5. To Check if all occupancies are evacuated or not, $\mathrm{K}$ ''s needs to be calculated as follow:

1. $\mathrm{K}$ 's $=\mathrm{Ks}-\mathrm{Cp}$ (selected paths) $=7-6=1$.

2. While K', $>0$.

3. $\mathrm{Tp}$ for $\mathrm{Ps}(1)=\mathrm{Tp}+\mathrm{tp}=15+1=16$.

4. $\mathrm{Tp}$ for $\mathrm{Ps}(3)=\mathrm{Tp}+\mathrm{tp}=15+1=16$.

5. $\mathrm{K}^{\prime} \mathrm{s}=\mathrm{Ks}-\mathrm{Cp}=7-6=1$.

6. $\mathrm{Ks}=\mathrm{K}$ 's $=1$.

7. Go back to step 5 , taking in considerations the new parameter values.

Iteration 3: Where, $\mathrm{Ks}<\mathrm{Cp}, \mathrm{wp}=\mathrm{Tp} / \mathrm{K}$ 's.

The values of Tp and wp for each path (Ps) is shown in Table 5.

Table 5. The values of Tp and wp for each path

\begin{tabular}{ccc}
\hline \multicolumn{1}{c}{ Path (Ps) } & Tp & wp \\
\hline Ps (1) = N1, N3, N4, N6, N10, N13. & 16 & 16 \\
Ps (2) = N1, N3, N4, N6, N10, N12, N14. & 20 & 20 \\
Ps (3) = N1, N3, N5, N7, N11, N14. & 16 & 16 \\
\hline
\end{tabular}


The Best Evacuation Path (BEP) is selected based on the minimum waiting time from all paths and they are Ps (1) and Ps (3) with a waiting time of 16. Due to the equivalence in the waiting time of Ps (1) and Ps (3), it is optional to choose any of them. As a result of evacuating all occupancies, the algorithm process will end. The total time needed to evacuate all occupancies in N1 is the summation of Tp for all iterations which is equal to 45 as shown in Table 6.

The presented steps of DRTCCR algorithm are applied on the remaining source nodes, N2 and N8 in the same way in N1. The total time needed to evacuate all occupancies in N2 is the summation of Tp for all iterations which is equal 29 as shown in Table 7. The total time needed to evacuate all occupancies in N8 is the summation of Tp for all iterations which is equal 14 as shown in Table 8.

The final evacuation plan for the selected transportation network model after applying the DRTCCR algorithm is shown in Table 9. The total time needed to evacuate all occupancies is 88 .

Table 6. The total evacuation time for N1

\begin{tabular}{cccc}
\hline Iterations & Selected Paths & $\begin{array}{c}\text { No. of } \\
\text { evacuees }\end{array}$ & $\begin{array}{c}\text { Evacuation } \\
\text { time }\end{array}$ \\
\hline Iteration No.1 & Ps (1) & 3 & 14 \\
Iteration No.2 & Ps (1) and Ps (3) & 6 & 15 \\
Iteration No.3 & Ps (1) & 1 & 16 \\
\hline
\end{tabular}

Table 7. The total evacuation time for N2

\begin{tabular}{cccc}
\hline Iterations & $\begin{array}{c}\text { Selected } \\
\text { Paths }\end{array}$ & $\begin{array}{c}\text { No. of } \\
\text { evacuees }\end{array}$ & $\begin{array}{c}\text { Evacuation } \\
\text { time }\end{array}$ \\
\hline Iteration No.1 & Ps (1) & 3 & 14 \\
Iteration No.2 & Ps (1) & 2 & 15 \\
\hline
\end{tabular}

Table 8. The total evacuation time for N8

\begin{tabular}{|c|c|c|c|}
\hline Iterations & $\begin{array}{l}\text { Selected } \\
\text { Paths }\end{array}$ & $\begin{array}{c}\text { No. of } \\
\text { evacuees }\end{array}$ & $\begin{array}{c}\text { Evacuation } \\
\text { time }\end{array}$ \\
\hline Iteration No.1 & Ps (1) & 6 & 4 \\
\hline Iteration No.2 & Ps (1) & 6 & 5 \\
\hline Iteration No.3 & Ps (6) & 3 & 5 \\
\hline
\end{tabular}

Table 9. The final evacuation plan for the selected transportation network model

\begin{tabular}{|c|c|c|c|}
\hline $\begin{array}{c}\text { Source } \\
\text { node }(\mathrm{Sn})\end{array}$ & Paths that used & $\begin{array}{c}\text { No. of } \\
\text { evacuees }\end{array}$ & $\begin{array}{c}\text { Evacuation } \\
\text { time }\end{array}$ \\
\hline \multirow{3}{*}{ For N1 } & Ps (1) & 3 & 14 \\
\hline & Ps (1) and Ps (3) & 6 & 15 \\
\hline & Ps $(1)$ & 1 & 16 \\
\hline \multirow{2}{*}{ For N2 } & Ps (1) & 3 & 14 \\
\hline & Ps (1) & 2 & 15 \\
\hline \multirow{3}{*}{ For N8 } & Ps (1) & 6 & 4 \\
\hline & Ps (1) & 6 & 5 \\
\hline & Ps (6) & 3 & 5 \\
\hline \multicolumn{2}{|r|}{ Total } & 30 & 88 \\
\hline
\end{tabular}

\section{RESEARCH METHOD}

The research method as well as the transportation network model with a building example are described and illustrated in this section. The research method can be formulated as shown in Figure 3. Transportation network defined as input, evacuation plan defined as output, as well as the objectives and constraints are illustrated.

The transportation network model is presented as a graph (i.e. nodes and edges) with capacity constraints, initial number of occupancy that should be evacuated, their initial locations and evacuation exits. The capacity constrained routing technique produces a set of routes to evacuate the occupancies. Consider a simple example as shown in Figure 4. Each node has two attributes, node capacity and initial node occupancy. For instance, the max capacity of node N1 is fifty persons, which means N1 can hold at most fifty persons with an initial occupancy of twenty, thus "the maximum allowed evacuees that can be moved out from N1 is twenty". In addition, every edge has two attributes: edge capacity and travel time. The arrows between any two nodes i.e. the maximum edge capacity for N1-N3 edge is five and this means that the maximum allowed evacuees number that can pass through this edge is five with a travel time equal three.

Dynamic real-time capacity constrained routing algorithm for evacuation planning... (Jawad Abusalama) 
Finally, assuming that N1 has twenty evacuees and is based on a capacity constrained routing approach, five evacuation routes can be generated as shown in Table 10. Thus, the objective of a capacity constrained routing approach is to minimize the total time needed for evacuation, as well as to minimize the computational cost of producing the evacuation plan.

Input (a transportation network)

- Source, destination and intermediate Nodes.

- Edges.

- Capacity constraints for each Node and Edge.

- Occupancies in each Source Nodes.

- Travel time for each Edge.

Objectives

To improve the performance by minimize the total time of evacuation, minimize the computational cost of producing the evacuation plan.

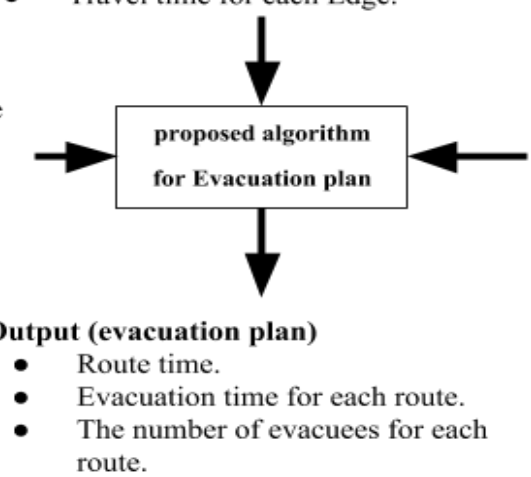

Constraints

Capacity constraints

of the transportation

network ( Nodes

and Edges capacity)

Figure 3. Research method

Table 10. Generated Evacuation Routes

\begin{tabular}{cccc}
\hline $\begin{array}{c}\text { Source } \\
\text { node }\end{array}$ & Evacuation Routs & $\begin{array}{c}\text { Destination } \\
\text { time }\end{array}$ & $\begin{array}{c}\text { Max } \\
\text { capacity }\end{array}$ \\
\hline N1 & N1-N2-N3-Exit1 & 9 & 8 \\
N1 & N1-N3-Exit1 & 4 & 5 \\
N1 & N1-N2-N4-Exit2 & 10 & 3 \\
N1 & N1-N4-Exit2 & 5 & 3 \\
N1 & N1-N3-N5-Exit2 & 12 & 3 \\
\hline
\end{tabular}
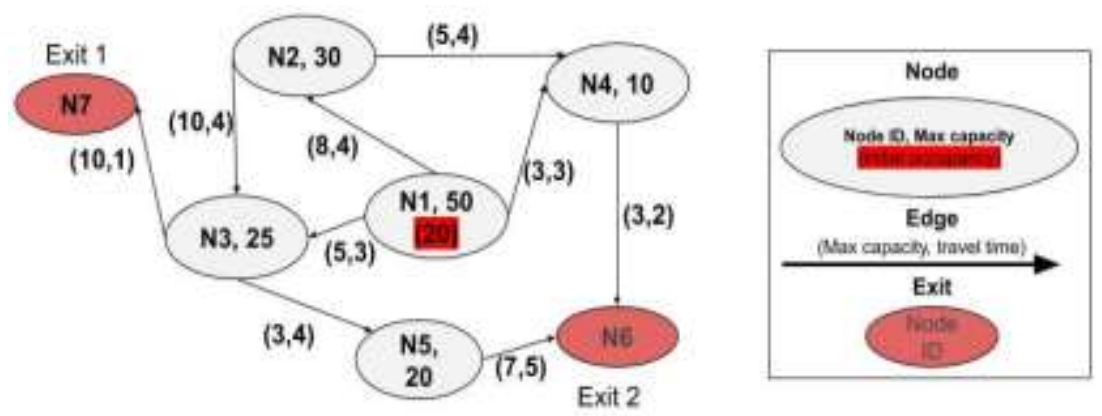

Figure 4. An example of transportation network

\section{RESULTS AND DISCUSSIONS}

The proposed algorithm has been analytically tested and evaluated by comparing it with other two previous algorithms. To validate such comparison, same transportation network model was used for each comparing operation. The first comparison was done between our proposed algorithm and the MRCCP algorithm proposed by Lu et al. [19], where he proposed and evaluated heuristic algorithms of the capacity constrained routing approach to find a suitable evacuation plan. The authors applied their proposed algorithm on the transportation network model which was shown above in Figure 2. The total evacuation time of 
MRCCP was equal 108. On the other hand, the total evacuation time for our proposed plan after applying it on the same transportation network model is equal 88, which means that the DRTCCR is $20 \%$ faster than MRCCP, and Figure 5 illustrates the comparison results between MRCCP and DRTCCR.

The second comparison was done between our proposed algorithm and the Max-Flow Rate Priority (MFRP) algorithm, which was proposed by Guo et al. [17], where the author proposed a recent heuristic approach to solve the evacuation route planning problem with a capacity constrained transportation network to find a suitable evacuation plan. He applied their proposed algorithm on the transportation network model, and the total evacuation time of the (MFRP) algorithm is equal 92, while the total evacuation time for our proposed plan is equal 53, which infers that DRTCCR is faster than MFRP by 39\%. Figure 6 illustrates the comparison results between DRTCCR and MFRP.

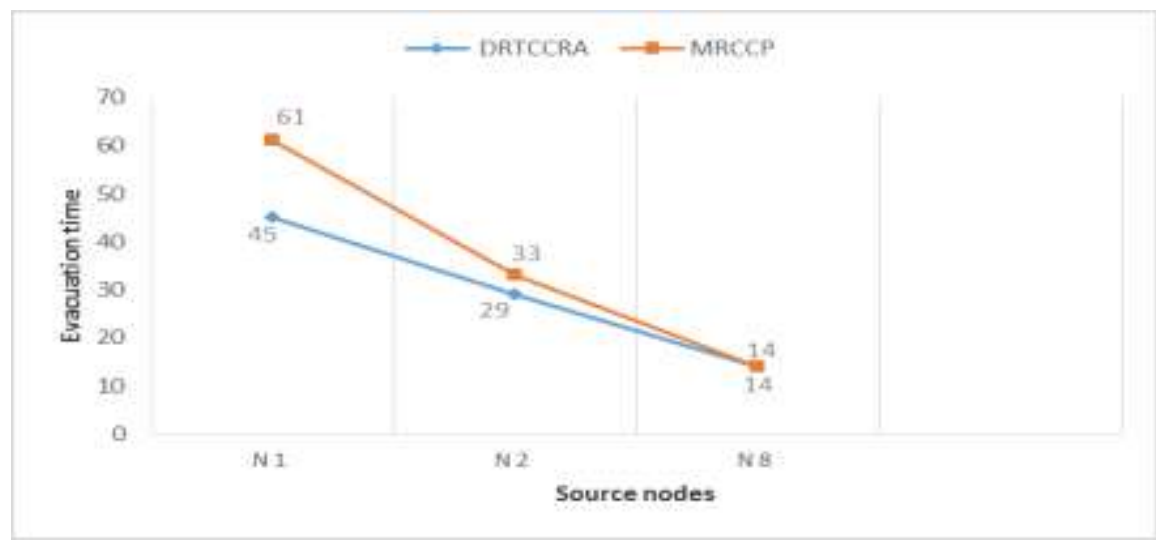

Figure 5. Comparison results between DRTCCR and MRCCP

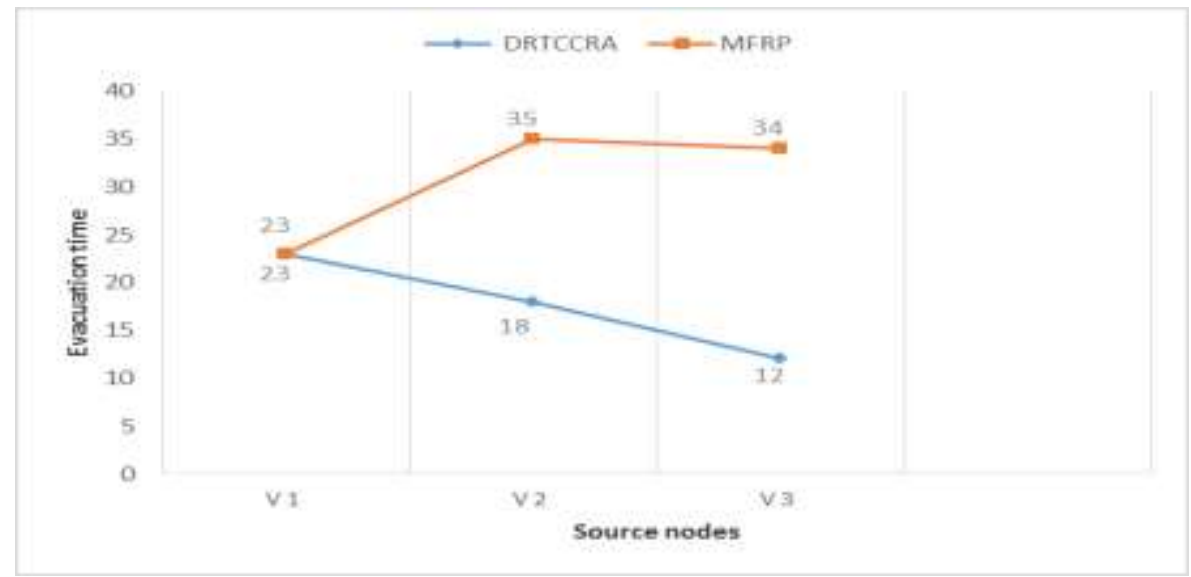

Figure 6. Comparison results between DRTCCR and MFRP

\section{CONCLUSION}

One of the most important challenges during evacuation in case of the occurrence of a disaster is the difficulty of finding the best and fastest paths to save the people at risk. In this paper, a dynamic real-time capacity constrained routing (DRTCCR) algorithm is proposed, tested and evaluated. Such algorithm will be used to improve the performance of an evacuation plan by minimizing the total evacuation time for all evacuees. Various network models were used to simulate such problems, coupled with the application of many evacuation planning algorithms on such models. Also, analytical and comparison studies were carried out and the result from both studies illustrates that the DRTCCR algorithm reduces the evacuation time compared with the MRCCP algorithm by $20 \%$, and by $39 \%$ when compared with the MFRP algorithm. To conclude, the proposed algorithm has proven to be efficient as compared to other related algorithms, in dealing with evacuation problems dynamically in real time. 


\section{ACKNOWLEDGEMENTS}

The author would like to thank the center for robotics \& industrial automation and the center for advanced computing technology in UTeM University, Melaka, Malaysia.

\section{REFERENCES}

[1] V. Campos, et al., "A Method for Evacuation Route Planning in Disaster Situations," Procedia - Soc. Behav. Sci., vol. 54, no. 4, pp. 503-512, 2012.

[2] J. Abusalama, et al., "A Review on Disaster Management System Based on Multi Agent Approaches," JARDCS, vol. Special Is, no. 07, pp. 721-731, 2018.

[3] M. Nor, et al., "Dynamic crowd evacuation approach for the emergency route planning problem: Application to case studies Dynamic crowd evacuation approach for the emergency route planning problem: Application to case studies," Saf. Sci., vol. 102, pp. 263-274, 2018.

[4] J. Abusalama, et al., "An Early Disaster Detection, Evacuation and Rescuing System Based on Multi-Agents Approach,” JARDCS, vol. Special Issue, no. 07, pp. 712-720, 2018.

[5] F. Horita, et al., "Understanding the decision-making process in disaster risk monitoring and early-warning: A case study within a control room in Brazil," Int. J. Disaster Risk Reduct., vol. 28, pp. 22-31, 2018.

[6] S. Shekhar, et al., "Experiences with evacuation route planning algorithms," International Journal of Geographical Information Science, vol. 26, no. 12. pp. 2253-2265, 2012.

[7] J. Cochran, et al., "Wiley Encyclopedia of Operations Research and Management Science," John Wiley \& Sons, pp. 210-212, 2011.

[8] A. Abdelghany, et al., "Modeling framework for optimal evacuation of large-scale crowded pedestrian facilities," Eur. J. Oper. Res., vol. 237, no. 3, pp. 1105-1118, 2014.

[9] S. R. Khadka and P. P. Bhandari, "Dynamic Network Contraflow Evacuation Planning Problem with Continuous Time Approach,” International Journal of Operations Research, vol. 14, no. 1, pp. 27-34, 2017.

[10] S. Alamri, "An Efficient Shortest Path Routing Algorithm for Directed Indoor Environments," ISPRS Int. J. GeoInformation, vol. 7, no. 4, p. 133, 2018.

[11] H. W. W. Hamacher and S. A. A. Tjandra, "Mathematical modelling of evacuation problems: state of the art," Pedestr. Evacuation Dyn, vol. 24, no. 24, pp. 227-266, 2002.

[12] M. Kisko and L. Francis, "EVACNET+: A computer program to determine optimal building evacuation plans," Fire Saf. J., vol. 9, no. 2, pp. 211-220, 1985.

[13] Z. Hu, et al., "Simulation of Optimized Evacuation Processes based on Sub-Goal CA Model," International Journal of Grid and Distributed Computing, vol. 9, no. 5, pp. 133-144, 2016.

[14] R. Xie and L. Li, "Simulation of Optimized Evacuation Processes in Complex Buildings Using Cellular Automata Model," Journal of software, vol. 9, no. 6, pp. 1428-1434, 2014.

[15] M. Khalid and U. Yusof, "An Artificial Immune Approach for Optimizing Crowd Emergency Evacuation Route Planning Problem," in Proceedings of the International Conference on Agents and Artificial Intelligence, Portugal, pp. 503-508, 2015.

[16] O. J. Akinwande, et al., "Managing Crowds in Hazards with Dynamic Grouping," IEEE Access, vol. 3, pp. 1060-1070, 2015.

[17] D. Guo, et al., "Max-Flow rate priority algorithm for evacuation route planning," Proc. - 2016 IEEE 1st Int. Conf. Data Sci. Cyberspace, pp. 275-283, 2016.

[18] Q. Lu, et al., "Capacity Constrained Routing Algorithms for Evacuation Planning: A Summary of Results," Lncs, vol. 3633, no. 81655, pp. 291-307, 2005.

[19] Q. Lu, et al., "Evacuation Planning: A Capacity Constrained Routing Approach," in Proceedings of the First NSF/NIJ Symposium on Intelligence and Security Informatics, USA, pp. 111-125, 2003.

[20] Z. Fang, et al., "A space-time efficiency model for optimizing intra-intersection vehicle-pedestrian evacuation movements," Transp. Res. Part C Emerg. Technol., vol. 31, pp. 112-130, 2013.

[21] G. J. Lim, et al., "A capacitated network flow optimization approach for short notice evacuation planning," Eur. J. Oper. Res., vol. 223, no. 1, pp. 234-245, 2012.

[22] M. Zeng and C. Wang, "Evacuation Route Planning Algorithm: Longer Route Preferential," Adv. Neural Networks, vol. 5551, pp. 1062-1071, 2009.

[23] S. Al-Khammasi, et al., "Energy Efficient Cluster Based Routing Protocol for Dynamic and Static Nodes in Wireless Sensor Network," TELKOMNIKA Telecommunication Computing Electronics and Control, vol. 16, no. 5, pp. 1974-1981, 2018.

[24] D. T. Do, et al., "Wireless power transfer enabled NOMA relay systems: two SIC modes and performance evaluation," TELKOMNIKA Telecommunication Computing Electronics and Control, vol. 17, no 6, pp. 250-260, 2019.

[25] A. Lit, et al., "Comparative performance evaluation of routing algorithm and topology size for wireless network-onchip," Bulletin of Electrical Engineering and Informatics, vol. 8, no. 4, pp. 1239-1250, 2019. 